\title{
Doppler-free Fourier transform spectroscopy
}

\author{
Samuel A. Meek, ${ }^{1}$ Arthur Hipke, ${ }^{1,2}$ Guy Guelachvili, ${ }^{3}$ Theodor W. Hänsch, ${ }^{1,2}$ and \\ Nathalie Picqué ${ }^{1,2,3, *}$ \\ ${ }^{1}$ Max-Planck-Institut für Quantenoptik, Hans-Kopfermann-Straße 1, 85748 Garching, Germany \\ ${ }^{2}$ Ludwig-Maximilians-Universität München, Fakultät für Physik, Schellingstr. 4/III, 80799 München, Germany \\ ${ }^{3}$ Institut des Sciences Moléculaires d'Orsay (ISMO), CNRS, Univ. Paris-Sud, Université Paris-Saclay, F-91405 Orsay, France \\ *Corresponding author: nathalie.picque@mpq.mpg.de
}

Received 20 October 2017; revised 24 November 2017; accepted 25 November 2017; posted 29 November 2017 (Doc. ID 308719 );

published 22 December 2017

\begin{abstract}
Sub-Doppler broadband multi-heterodyne spectroscopy is proposed and experimentally demonstrated. Using two laser frequency combs of slightly different repetition frequencies, we have recorded Doppler-free two-photon dual-comb spectra of atomic rubidium resonances of a width of $6 \mathrm{MHz}$, while simultaneously interrogating a spectral span of $10 \mathrm{THz}$. The atomic transitions are uniquely identified via the intensity modulation of the observed fluorescence radiation. To the best of our knowledge, these results represent the first demonstration of Doppler-free Fourier transform spectroscopy and extend the range of applications of broadband spectroscopy towards precision nonlinear spectroscopy. (־) 2017 Optical Society of America
\end{abstract}

OCIS codes: (300.6300) Spectroscopy, Fourier transforms; (300.6420) Spectroscopy, nonlinear.

https://doi.org/10.1364/OL.43.000162

Provided under the terms of the OSA Open Access Publishing Agreement

For nearly 50 years, Fourier transform spectroscopy has been the leading technique in analytical chemistry and molecular spectroscopy, as well as an irreplaceable tool for remote sensing, astronomy, industrial process control, etc. On a single photodetector and in almost any spectral region, Fourier transform spectrometers record multiplex high-resolution spectra over a broad spectral span. Interferometers with a resolution better than $30 \mathrm{MHz}$ are available [1,2], even commercially; however, high-resolution Fourier transform spectroscopy in the gas phase has been so far limited to the measurements of Dopplerbroadened lines, possibly narrowed by cooling the sample, e.g., in a supersonic beam or by collisions with a buffer gas [3]

Nonlinear mechanisms for canceling the Doppler effect have been harnessed with tunable lasers or with a single frequency comb. In direct frequency comb Doppler-free two-photon spectroscopy [4-6], all comb lines may contribute, and the excitation probability of any given level can be the same as with a continuous-wave (cw) laser of the same average power. Short pulses facilitate nonlinear frequency conversion to access spectral regions where $\mathrm{cw}$ lasers are not readily available. However, the spectrum is only retrieved modulo the comb line spacing. Therefore, direct frequency comb spectroscopy with a single frequency comb is only suitable for spectra composed of very few transitions $[4,6-8]$, although coherent control enables the excitation of different transitions at distinct spatial positions [9].

Here we propose and demonstrate Doppler-free Fourier transform spectroscopy by combining dual-comb spectroscopy and two-photon excitation in a standing-wave field. Dual-comb spectroscopy is a recent technique of Fourier spectroscopy without moving parts, where the time-domain interference between two combs of slightly different repetition frequencies is recorded. Mostly exploited for linear absorption spectroscopy, first proof-of-principle experiments have extended its potential to nonlinear phenomena [10-15]. Our new technique enhances such capabilities by combining, for the first time, to the best of our knowledge, sub-Doppler resolution and a broad spectral range with a single photodetector.

We first give a simple explanation (Fig. 1) of the principle of Doppler-free two-photon excitation dual-comb spectroscopy in both the time and frequency domains.

In the time domain [Fig. 1(a)], the atomic (or molecular) sample is excited by two pairs of counter-propagating pulse trains of slightly different repetition frequencies $\left(f_{\mathrm{r}}\right.$ and $\left.f_{\mathrm{r}}+\Delta f_{\mathrm{r}}\right)$ in a standing-wave configuration, forming two trains of standing-wave pulses. For simplicity, we first assume that only one transition is excited and that the coherence time of the transition is sufficiently short to consider excitation by only two consecutive standing-wave pulses, one from each laser. The first standing-wave pulse excites the atoms into a coherent superposition of ground state and excited state, thus setting the origin of phase. The resulting atomic coherence precesses at the atomic resonance frequency $f_{\mathrm{at}}$, which is proportional to the difference in level energies. Then the second standing-wave pulse excites the sample at a time delay $T$ which increases from pulse pair to pulse pair by increments $\tau=1 / f_{\mathrm{r}}-1 /\left(f_{\mathrm{r}}+\Delta f_{\mathrm{r}}\right) \approx$ $\Delta f_{\mathrm{r}} / f_{\mathrm{r}}^{2}$. Because of the a-synchronism of the two pulse trains, this time delay is automatically scanned. The probability of having the atom in the excited state after the second pulse depends on the relative phase of the second standing-wave pulse to the phase of the atomic coherence oscillating with the resonance period $1 / f_{\mathrm{at}}$. Constructive interference between the two excitations occurs when $T$ equals an integer multiple of 


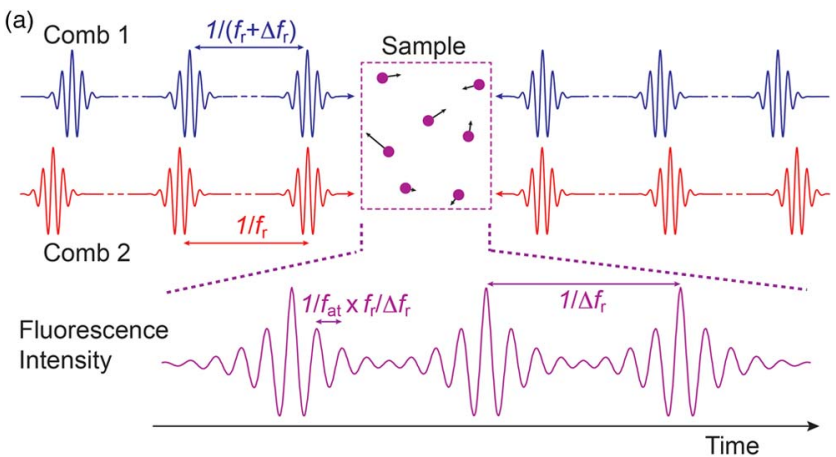

(b)

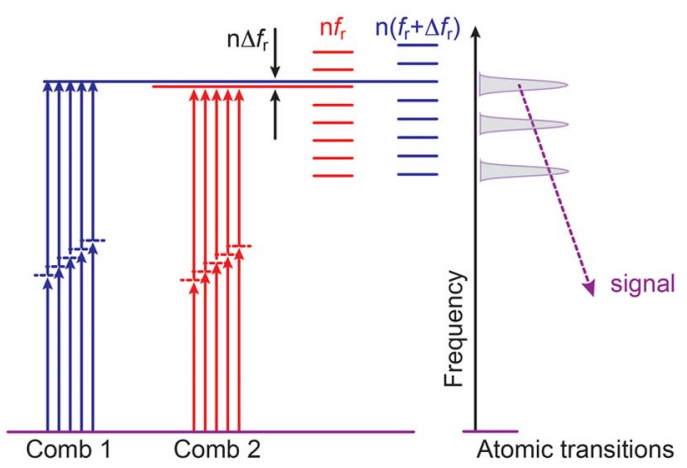

Fig. 1. Principle of Doppler-free two-photon excitation dual-comb spectroscopy. See also the text for details. (a) Time-domain picture. The atomic sample is excited by two pairs of counter-propagating pulse trains of slightly different periods in a standing-wave configuration, forming two trains of standing-wave pulses. The upper-state population is modulated as a function of the variable time delay between two pulses from the two asynchronous sources. The modulation period is proportional to the atomic resonance period and can be observed as an intensity modulation in the fluorescence. (b) Frequency-domain picture. When two frequency combs of slightly different repetition frequencies simultaneously excite a two-photon transition, an intensity modulation that unambiguously identifies the optical frequency of the transition is observed in the fluorescence during decays to lower states.

the atomic period $1 / f_{\mathrm{at}}$. As $T$ changes, the observable atomic fluorescence signal is modulated with a time period $1 / f_{\text {at }} \times$ $f_{\mathrm{r}} / \Delta f_{\mathrm{r}}$ or a frequency $f_{\mathrm{at}} \times \Delta f_{\mathrm{r}} / f_{\mathrm{r}}$. The interference signal decays with a time constant proportional to the lifetime of the upper state (or with a time constant induced by systematic instrumental effects, such as time-of-flight broadening due to the limited overlap volume for counter-propagating ultra-short pulses). The time-domain signal recurs with a period of $1 / \Delta f_{\mathrm{r}}$. If several transitions are simultaneously excited, the time-domain fluorescence signal, the interferogram, is the sum of the individual interference terms, each oscillating at a uniquely defined frequency proportional to its respective atomic resonance frequency. The Fourier transform of the interferogram is the Doppler-free two-photon excitation spectrum, sampled by the frequency comb discrete lines. Our technique with an automatic scan of the time delay between the pulse pairs is an extension of the optical analog of the Ramsey-fringe method [16,17]: several transitions may be simultaneously and unambiguously observed and assigned.

In the frequency domain [Fig. 1(b)], a frequency comb excites a two-photon transition when the sum of two comb line frequencies is in resonance with the atomic line profile. Many pairs of comb modes have the same sum frequency, so that the excitation probability can be the same as for a resonantly tuned $\mathrm{cw}$ laser of the same average power [4-6]. The excited line profile is the intrinsic atomic (or molecular) line shape sampled by the resonant sum frequency lines of the frequency comb. When the excitation of a line of frequency $f_{\mathrm{at}}$ close to $\mathrm{n} f_{\mathrm{r}}$, (where $\mathrm{n}$ is an integer) is performed by two mutually-coherent frequency combs of slightly different repetition frequencies $f_{\mathrm{r}}$ and $f_{\mathrm{r}}+\Delta f_{\mathrm{r}}$, the probability of excitation of the transition by the two combs includes an interference term oscillating at the frequency $\mathrm{n} \Delta f_{\mathrm{r}}$. The modulation at the radio-frequency $\mathrm{n} \Delta f_{\mathrm{r}}$ uniquely identifies the excited optical transition. This modulation, for instance, can be monitored through the variations of the intensity of the fluorescence of the upper level during decays toward lower states. The resulting two-photon excitation spectrum has all the characteristics of a dual-comb (or multiheterodyne) spectrum: the free-spectral range is only limited by the span of the exciting laser combs. The resolution in a spectrum is determined by the comb line spacing. The position of each comb line may be known within the accuracy of an atomic clock, and the frequency scale may be directly selfcalibrated. Since the signal modulation frequency is obtained from the interference of two frequency combs, in principle, it would be advantageous to work with a very small comb line spacing, e.g., $1 \mathrm{MHz}$. If the line spacing is smaller than the width of the atomic transition, the simplistic two-pulse picture in the time domain becomes valid. Such a scenario would be of particular interest for experiments with amplified laser systems, although the stringent stability requirements render their prospect daunting. Alternatively, one can use oscillators of conventional repetition frequency (around $100 \mathrm{MHz}$ ). The position of the comb lines may then be scanned in a step-wise manner, and several spectra may be interleaved to improve the resolution, possibly down to the optical comb linewidth. Intuitively, this is understandable as the Fourier transform is a linear transform. In the time domain, many pulses contribute to coherently build up the atomic probability amplitude, and the time delay between the pulse pairs should vary negligibly during this buildup time. As the observed interference signal is generated by a modulation of the population of the upper state, the lifetime of this state acts as a low-pass filter on the interferometric modulation. Therefore, the radio-frequency free spectral range may be determined [12] by the natural linewidth of the transitions, rather than by half the comb repetition frequency.

We experimentally demonstrate our technique with the two-photon excitation spectrum of the 5S-5D transitions of rubidium vapor around $2 \times 385 \mathrm{THz}$. The setup (Fig. 2) builds on that developed for Doppler-limited two-photon excitation spectroscopy [12] with a modified optical layout that allows standing-wave excitation of the sample with counterpropagating beams.

Two frequency-doubled erbium-doped fiber laser comb generators emit around $383 \mathrm{THz}\left(783 \mathrm{~nm}, 12775 \mathrm{~cm}^{-1}\right)$ with a repetition frequency of about $100 \mathrm{MHz}$ and an adjustable difference in repetition frequencies $\Delta f_{\mathrm{r}}$. They span about $10 \mathrm{THz}$. The repetition frequencies are stabilized against the radio-frequency signal of a hydrogen maser, as all electronics in the setup. One of their comb lines is stabilized to a $193 \mathrm{THz}$ narrow-linewidth $\mathrm{cw}$ laser. The mode numbers and the width (narrower than $1 \mathrm{kHz}$ at $1 \mathrm{~s}$ ) of the optical comb 


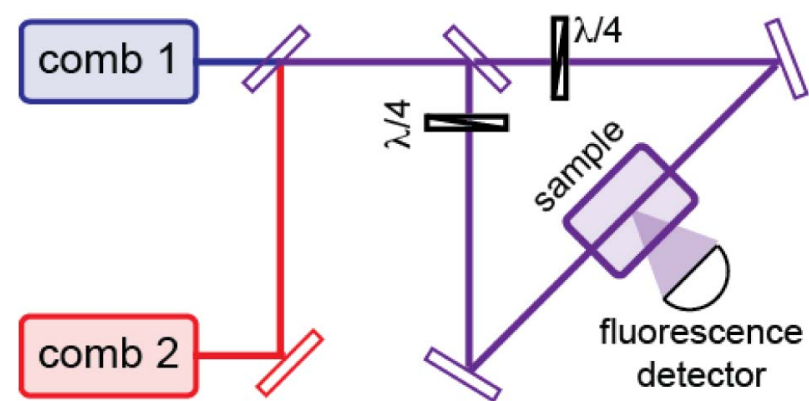

Fig. 2. Experimental setup for Doppler-free dual-comb spectroscopy with two-photon excitation.

lines are measured using an out-of-loop narrow-linewidth cw laser around $195 \mathrm{THz}$. The cw lasers are referenced to a metrological frequency comb, self-referenced to the maser. The beams of the two frequency-doubled combs are combined before entering an anti-resonant ring, which contains a magnetically shielded rubidium cell, heated to $320 \mathrm{~K}$. The two counter-propagating beams are circularly polarized with the same sense of rotation. They are focused at the same position of the cell, and the total average power at the focus is about $10 \mathrm{~mW}$, balanced between the four contributing comb beams (two in each direction). The fluorescence at $710 \mathrm{THz}(420 \mathrm{~nm}$, $23683 \mathrm{~cm}^{-1}$ ), induced by the two-photon excitation, is collected with a photomultiplier. The modulations of the fluorescence intensity provide the time-domain interference signal, recorded on a fast digitizer. The recording time per interferogram is $36 \mathrm{~s}$. An a-posteriori correction scheme [12] corrects the interferograms for the residual relative fluctuations of the combs. Each interferogram is Fourier transformed, and the spectral samples corresponding to radio-frequency comb line positions are sorted out. To improve the resolution, limited in one spectrum by the comb line spacing of $100 \mathrm{MHz}$, we interleave 100 spectra, each recorded by shifting the positions of the optical comb lines. As a result, we sample the Dopplerfree line profiles of the two-photon transitions around $770 \mathrm{THz}$ $\left(389 \mathrm{~nm}, 25684 \mathrm{~cm}^{-1}\right)$ with an effective resolution of $1 \mathrm{MHz}\left(3 \times 10^{-5} \mathrm{~cm}^{-1}\right)$.

The radio-frequency free spectral range is limited [12] by the upper-state lifetime to about $2 \mathrm{MHz}$. In a first experiment, 100 interferograms of $537 \times 10^{6}$ samples each, with $\Delta f_{\mathrm{r}}=10 \mathrm{~Hz}$, are interleaved, leading to a spectrum of $1-\mathrm{MHz}$ resolution. The free spectral range is twice as broad as the span [Fig. 3(a)] of $10 \mathrm{THz}$ where Doppler-free transitions may be excited by the dual-comb system. The 5S-5D transitions of ${ }^{85} \mathrm{Rb}$ and ${ }^{87} \mathrm{Rb}$ in natural abundance [Fig. 3(a)] only cover $100 \mathrm{GHz}$. In the next recording, we record interferograms of $67 \times 10^{6}$ samples each, with $\Delta f_{\mathrm{r}}=380 \mathrm{~Hz}$ to speed up the data processing. Therefore, the free spectral range is limited to $0.5 \mathrm{THz}$, still significantly broader than the required span of $100 \mathrm{GHz}$ [Fig. 3(b)]. In contrast, conventional direct frequency comb excitation with a single frequency comb folds (Fig. 4) all these transitions into a free spectral range of $100 \mathrm{MHz}$. In Fig. 3, at the pedestal of the intense $5 \mathrm{~S}_{1 / 2}-5 \mathrm{D}_{5 / 2}$ lines around $770.57 \mathrm{THz}$, the residual Doppler profile coming from traveling-wave excitation is distinguishable. The signal-to-noise ratio of the most intense transition $\left(5 \mathrm{~S}_{1 / 2} F=3-5 \mathrm{D}_{5 / 2}\right)$ of ${ }^{85} \mathrm{Rb}$ is higher than 60,000 . The good signal-to-noise ratio is a consequence of the efficient

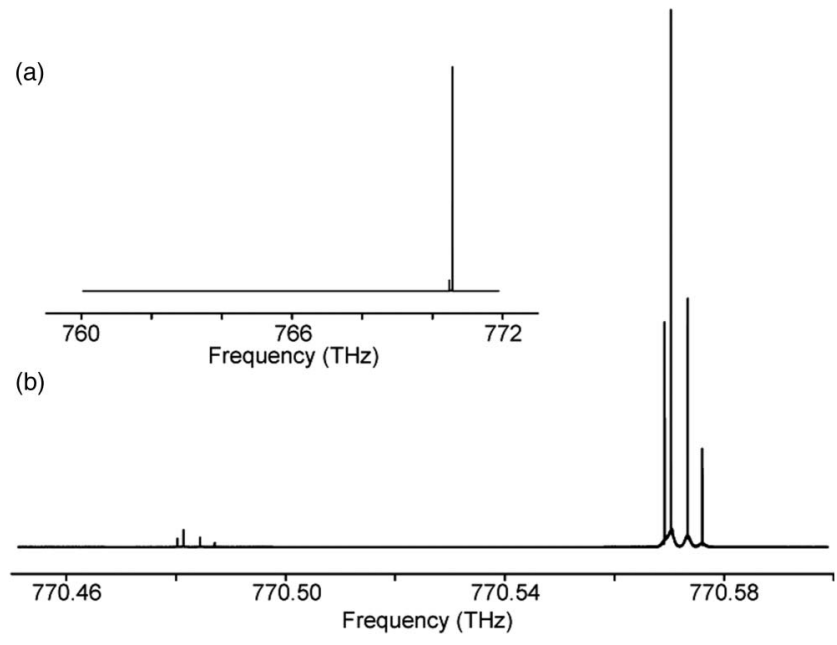

Fig. 3. Experimental dual-comb two-photon spectra of the subDoppler hyperfine transitions of ${ }^{85} \mathrm{Rb}$ and ${ }^{87} \mathrm{Rb}$. (a) The span is broader than $10 \mathrm{THz}$. (b) The lines around $770.48 \mathrm{THz}$ are assigned to the $5 \mathrm{~S}_{1 / 2}-5 \mathrm{D}_{3 / 2}$ transitions, and those around $770.57 \mathrm{THz}$ are assigned to the $5 \mathrm{~S}_{1 / 2}-5 \mathrm{D}_{5 / 2}$ transitions.

excitation scheme, where many pairs of comb lines contribute, and of the background-free fluorescence detection. Figure 5 provides a magnified representation of the $5 \mathrm{~S}_{1 / 2} F=2-5 \mathrm{D}_{3 / 2}$ $F=0,1,2,3$ hyperfine transitions of ${ }^{87} \mathrm{Rb}$ and of the $5 \mathrm{~S}_{1 / 2}$ $F=2-5 \mathrm{D}_{5 / 2} F=4,3,2,1$ hyperfine transitions of ${ }^{87} \mathrm{Rb}$. The observed width of the lines is $6 \mathrm{MHz}\left(2 \times 10^{-4} \mathrm{~cm}^{-1}\right)$, due to the time-of-flight broadening. The frequency scale is directly referenced to the hydrogen maser. The experimental absolute transition frequencies of the non-blended lines are found to agree within $200 \mathrm{kHz}$ with the literature. We attribute the main source of uncertainty to frequency pulling effects due to the absorption of the comb light by the intermediate 5P state before the excitation focal point in the cell. A detailed assessment of the systematic effects in our technique is beyond the scope of the present report, and it will be performed in the near future with

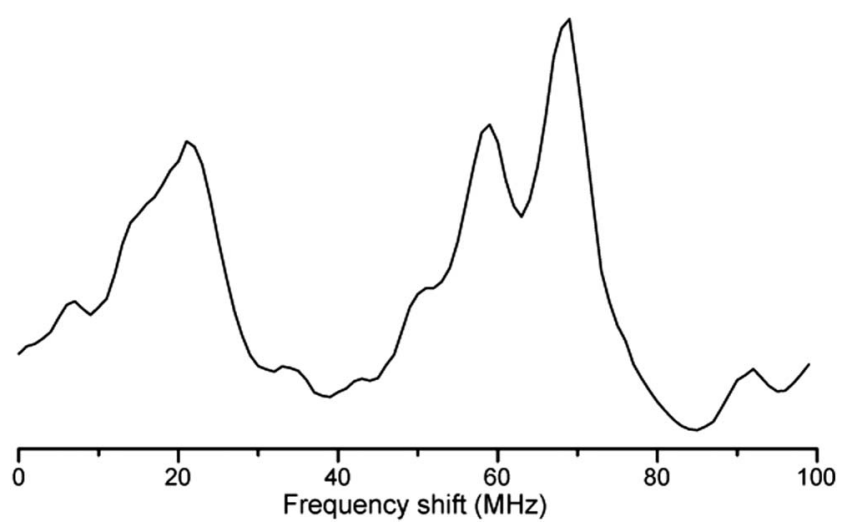

Fig. 4. Spectrum of Doppler-free two-photon spectroscopy with a single frequency comb of $100 \mathrm{MHz}$ line spacing. All the $5 \mathrm{~S}_{1 / 2}-5 \mathrm{D}_{3 / 2}$ and $5 \mathrm{~S}_{1 / 2}-5 \mathrm{D}_{5 / 2}$ transitions of ${ }^{85} \mathrm{Rb}$ and ${ }^{87} \mathrm{Rb}$ are simultaneously excited, and they appear blended and folded into a free spectral range of $100 \mathrm{MHz}$, whereas the corresponding dual-comb spectrum (Fig. 3) of the same sample unambiguously resolves the optical transitions. 


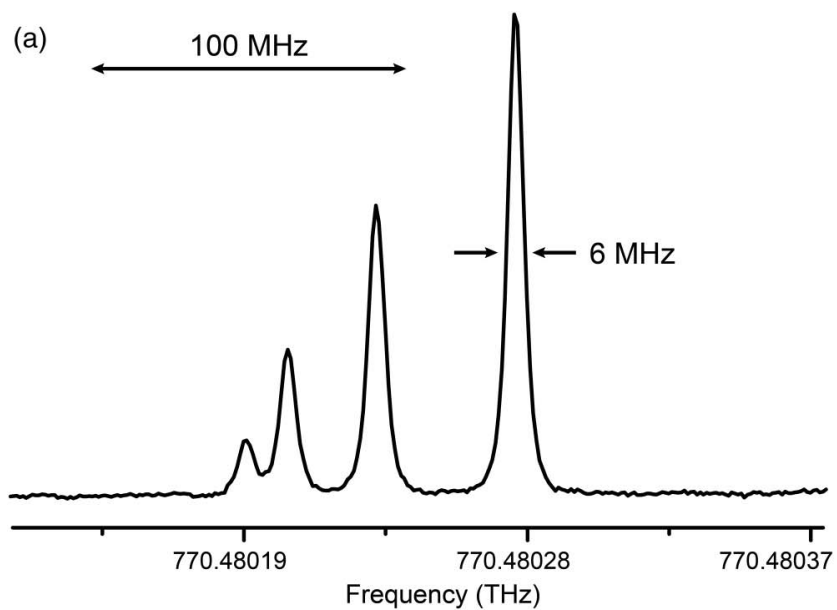

(b)

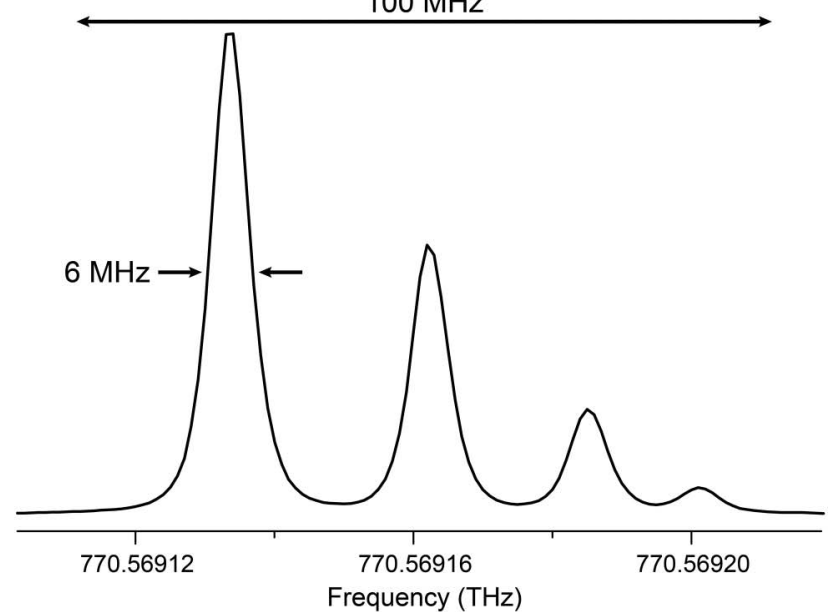

Fig. 5. Magnified views of the experimental sub-Doppler dualcomb two-photon spectrum shown in Fig. 3(b). The observed full-width at half-maximum of the isolated lines is $6 \mathrm{MHz}$. The $100-\mathrm{MHz}$ span in the upper part of each panel indicates the free spectral range that would be obtained with the established technique of direct frequency comb two-photon spectroscopy with a single frequency comb. (a) The lines are assigned (in ascending frequency) to the $5 \mathrm{~S}_{1 / 2} F=2-5 \mathrm{D}_{3 / 2} F=0,1,2,3$ transitions of ${ }^{87} \mathrm{Rb}$. The signal-to-noise ratio of the $5 \mathrm{~S}_{1 / 2} F=2-5 \mathrm{D}_{3 / 2} F=3$ hyperfine line is 920. (b) The lines, which frequency is about $89 \mathrm{GHz}$ apart from those shown in (a), are assigned to the $5 \mathrm{~S}_{1 / 2} F=2-5 \mathrm{D}_{5 / 2}$ $F=4,3,2,1$ transitions of ${ }^{87} \mathrm{Rb}$. The signal-to-noise ratio of the $5 \mathrm{~S}_{1 / 2} F=2-5 \mathrm{D}_{5 / 2} F=4$ hyperfine line is 25,000 .

better-suited transitions in a more controlled sample environment. The experiments summarized above are, to the best of our knowledge, the first report [18] of broadband partly multiplex Doppler-free spectroscopy.

For truly multiplex measurements, one would have to use lasers with a line spacing equal to the desired spectral resolution. Small comb line spacing, or low repetition frequencies, could take advantage of amplified laser systems. As an additional benefit, their high pulse energy would facilitate the nonlinear frequency conversion of the exciting light in the mid-infrared or ultraviolet (UV) spectral region and the nonlinear excitation of the sample. A significant challenge, especially in the UV range, may be to achieve narrow comb linewidth and high mutual coherence with amplified systems. Feed-forward relative stabilization might provide solutions [19]. The prospect of using amplified systems for dual-comb spectroscopy is consistent with the path followed with narrowband Ramsey comb spectroscopy [20] and is opposite that of direct frequency comb two-photon spectroscopy with a single comb of a high repetition frequency, for which state-of-the-art systems still lack sufficient power [21]. Our new technique might open novel approaches to precision two-photon spectroscopy in the UV or even in the extreme ultraviolet. More generally, the combination of a wide spectral bandwidth and sub-Doppler resolution may enable broadband molecular spectroscopy with unprecedented precision.

Funding. H2020 European Research Council (ERC) (267854); Munich-Centre for Advanced Photonics (MAP); Max-Planck Foundation.

\section{REFERENCES}

1. G. Guelachvili, Appl. Opt. 16, 2097 (1977).

2. S. Albert, K. Keppler Albert, P. Lerch, and M. Quack, Faraday Discuss. 150, 71 (2011).

3. B. Spaun, P. B. Changala, D. Patterson, B. J. Bjork, O. H. Heckl, J. M. Doyle, and J. Ye, Nature 533, 517 (2016).

4. R. Teets, J. Eckstein, and T. W. Hänsch, Phys. Rev. Lett. 38, 760 (1977).

5. Y. V. Baklanov and V. P. Chebotayev, Appl. Phys. 12, 97 (1977)

6. J. N. Eckstein, A. I. Ferguson, and T. W. Hänsch, Phys. Rev. Lett. 40, 847 (1978).

7. E. Peters, D. C. Yost, A. Matveev, T. W. Hänsch, and T. Udem, Annalen der Physik 525, L29 (2013).

8. A. Ozawa and Y. Kobayashi, Phys. Rev. A 86, 022514 (2012).

9. I. Barmes, S. Witte, and K. S. E. Eikema, Nat. Photonics 7, 38 (2013).

10. T. Ideguchi, B. Bernhardt, G. Guelachvili, T. W. Hänsch, and N. Picqué, Opt. Lett. 37, 4498 (2012).

11. T. Ideguchi, S. Holzner, B. Bernhardt, G. Guelachvili, N. Picqué, and T. W. Hänsch, Nature 502, 355 (2013).

12. A. Hipke, S. A. Meek, T. Ideguchi, T. W. Hänsch, and N. Picqué, Phys. Rev. A 90, 011805(R) (2014).

13. K. J. Mohler, B. J. Bohn, M. Yan, G. Mélen, T. W. Hänsch, and N. Picqué, Opt. Lett. 42, 318 (2017).

14. A. Asahara and K. Minoshima, APL Photon. 2, 041301 (2017).

15. B. Lomsadze and S. T. Cundiff, Opt. Lett. 42, 2346 (2017).

16. N. F. Ramsey, Phys. Rev. 78, 695 (1950).

17. M. M. Salour and C. Cohen-Tannoudji, Phys. Rev. Lett. 38, 757 (1977).

18. A. Hipke, S. A. Meek, G. Guelachvili, T. W. Hänsch, and N. Picqué, Conference on Lasers and Electro Optics (CLEO), OSA Postdeadline Paper Digest (online) (Optical Society of America, 2013), paper CTh5C.8.

19. Z. Chen, M. Yan, T. W. Hänsch, and N. Picqué, "A phase-stable dual-comb interferometer," arXiv:1705.04214 (2017).

20. R. K. Altmann, S. Galtier, L. S. Dreissen, and K. S. E. Eikema, Phys. Rev. Lett. 117, 173201 (2016).

21. A. Cingöz, D. C. Yost, T. K. Allison, A. Ruehl, M. E. Fermann, I. Hartl, and J. Ye, Nature 482, 68 (2012). 